\title{
THE PICARD-VESSIOT CLOSURE IN DIFFERENTIAL GALOIS THEORY
}

\author{
ANDY R. MAGID \\ Department of Mathematics, University of Oklahoma \\ Norman, OK 73019, U.S.A. \\ E-mail: amagid@aftermath.math.ou.edu
}

1. Introduction. We begin with some notation and definitions:

$F$ denotes a differential field of characteristic zero with derivation $D=D_{F}$ and algebraically closed field of constants $C$.

$E \supset F$ is a Picard-Vessiot, or Differential Galois extension for an order $n$ monic linear homogeneous differential operator

$$
L=Y^{(n)}+a_{n-1} Y^{(n-1)}+\ldots+a_{1} Y^{(1)}+a_{0} Y, \quad a_{i} \in F
$$

if:

1. $E$ is a differential field extension of $F$ generated over $F$ by $V=\{y \in E \mid L(y)=0\}$.

2. The constants of $E$ are those of $F$ ("no new constants").

3. $\operatorname{dim}_{C}(V)=n$ ("full set of solutions").

For Picard-Vessiot extensions, let $G(E / F)=\operatorname{Aut}_{F}^{\text {diff }}(E)$; then $G(E / F) \rightarrow G L\left(L^{-1}(0)\right)$ is an injection with Zariski closed image.

There is a "Fundamental Theorem" for differential Galois extensions:

Theorem 1 (Fundamental Theorem for Picard-Vessiot Extensions). Let $E \supset F$ be a Picard-Vessiot extension. Then $G=G(E / F)$ has a canonical structure of affine algebraic group and there is a one-one lattice inverting correspondence between differential subfields $K, E \supset K \supset F$, and Zariski closed subgroups $H$ of $G$ given by $K \mapsto G(E / K)$ and $H \mapsto K^{H}$. If $K$ is itself a Picard-Vessiot extension, then the restriction map $G \rightarrow$ $G(K / F)$ is a surjection with kernel $G(E / K)$. If $H$ is normal in $G$, then $K^{H}$ is a PicardVessiot extension.

2000 Mathematics Subject Classification: Primary 12H05; Secondary 14L15, 20 G20.

Research supported by the NSF under grant number DMS 0070748.

The paper is in final form and no version of it will be published elsewhere. 
For various reasons, the direct analogues of "algebraic closure" and its properties for differential Galois extensions do not hold. However, the following notion is of interest:

A Picard-Vessiot closure $E \supset F$ of $F$ is a differential field extension which is a union of Picard-Vessiot extensions of $F$ and such that every such Picard-Vessiot extension of $F$ has an isomorphic copy in $E$.

ExAmple 1. For an example, we consider the Picard-Vessiot closure of $\mathbb{C}$ (with the trivial derivation). Let $\mathbb{C}[[t]]$ and $\mathbb{C}((t))$ denote the ring of complex formal power series and its quotient field, with the usual derivation $(D(\mathbb{C})=0$ and $D(t)=1)$. For $c \in \mathbb{C}$ we let $\exp (c t)=\sum(c t)^{k} / k$ !. Let $K$ be the subfield of $\mathbb{C}((t))$ generated over $\mathbb{C}$ by $t$ and $\{\exp (c t) \mid c \in \mathbb{C}\}$. Since $\exp (c t)$ is a solution of $Y^{\prime}-c Y=0$, it is easy to see that $\mathbb{C}(\exp (c t)) \supset \mathbb{C}$ is a Picard-Vessiot subextension of $\mathbb{C}((t)) \supset \mathbb{C}$. And since $t$ is a solution of $Y^{\prime \prime}=0, \mathbb{C}(t) \supset \mathbb{C}$ is a Picard-Vessiot subextension as well. As we will show below (Corollary 10$)$, this implies that the field $K$, the compositum of the fields $\mathbb{C}(\exp (c t))$, $c \in \mathbb{C}$, along with $\mathbb{C}(t)$, is a union of Picard-Vessiot subextensions of $\mathbb{C}$. On the other hand, it is a familiar fact from the theory of elementary differential equations that any linear, homogeneous equation with (complex) constant coefficients has a full set of solutions of the form $t^{k} \exp \left(c_{j} t\right)$ for appropriate $k$ and $c_{j} \in \mathbb{C}$. It follows that any Picard-Vessiot extension of $\mathbb{C}$ embeds in $K$, and hence that $K$ is a Picard-Vessiot closure of $\mathbb{C}$.

There are two basic approaches to the algebraic construction of Picard-Vessiot closures: one can either construct a maximal extension of a suitable sort by an application of Zorn's Lemma, and then try to prove that it contains copies of all Picard-Vessiot extensions of the base; or one can take a tensor product of all the Picard-Vessiot extensions of the base and then try to prove than an appropriate quotient exists. (Both approaches are related, of course.) In [2] and [3], we considered the construction from the first approach. (There are errors in the account in [2] which are corrected in [3].) In the present work, we follow the second approach, although we will use the first approach to establish the existence of the desired quotient. Our argument proceeds via a class of differential fields which are especially well adapted for the Zorn's lemma argument we need.

It should also be noted that these algebraic constructions are moot from a model theory point of view, where all the fields in question can already be assumed to reside inside a single universal differential field.

In [3], it is further shown that differential automorphisms of the base field lift to differential automorphisms of a Picard-Vessiot closure. We give another proof of that here, using the tensor product construction of closures, which makes this lifting theorem more transparent.

In [3], in addition to proving that Picard-Vessiot closures exist, it is shown that they have pro-affine proalgebraic groups of differential automorphisms, and that there is a "Fundamental Theorem" for Picard-Vessiot closures and related infinite extensions. (An interesting special case is the Picard-Vessiot antiderivative closure $E_{U} \supset F$ : the group $G$ of differential automorphisms of $E_{U}$ over $F$ is prounipotent. When $C$ is the complex numbers and $F=C(t)$ the rational functions in one variable, this $G$ is free prounuipotent.)

The Picard-Vessiot closure of $F$ can have proper Picard-Vessiot extensions, and hence a proper Picard-Vessiot closure. This leads naturally to the consideration of the tower 
of Picard-Vessiot extensions. The automorphism lifting theorem implies that the differential automorphism groups of each extension in the tower over the base maps onto the automorphism group of its predecessor, with the kernel being the pro-affine proalgebraic group of differential automorphisms of the extension over its predecessor. However the groups themselves need not be pro-affine.

2. Locally excellent fields. Our main interest is in differential fields which are unions of Picard-Vessiot extensions. For didactic purposes, however, we will work with a broader class of fields, ones for which we have extracted all the excellent properties of Picard-Vessiot extensions save normality. In keeping with a similar convention from commutative algebra, we will call such fields excellent. The following sequence of definitions sets the terminology.

Definition 1. A differential field extension $E \supseteq F$ is a $N o$ New Constants (NNC) extension if the constants of $E$ are those of $F$. A differential integral domain $R \supseteq F$ is called a No New Constants $F$ algebra if the quotient field $E$ of $R$ is NNC extension of $F$. A prime differential ideal $P$ of a differential $F$ algebra $S$ is called a co No New Constants (cNNC) ideal if $A / P$ is a NNC $F$ algebra.

It is shown, for example in [2], that if $A$ is a finitely generated differential $F$ algebra then any maximal differential ideal $M$ is a cNNC ideal.

Definition 2. An integral domain differential $F$ algebra $R$ is called excellent if:

1. Every element of $R$ satisfies a linear differential equation over $F$.

2. $R$ is a finitely generated $F$ algebra.

3. $R$ is a NNC $F$ algebra.

As noted, if a finitely generated differential $F$ algebra is differentially simple, then it is also NNC. For such algebras, condition 3 is superfluous. We call differentially simple excellent algebras simply excellent. A differential field extension $E \supseteq F$ is called excellent (respectively simply excellent) if it is the quotient field of an excellent (respectively simply excellent) differential $F$ algebra. $E$ is called locally excellent (respectively locally simply excellent, respectively locally Picard-Vessiot) if every finite subset of $E$ is contained in an excellent (respectively simply excellent, respectively Picard-Vessiot) subfield.

By [2, Thm 3.4 \& Thm 3.5, p. 25], a Picard-Vessiot extension of $F$ is simply excellent. It is easy to construct excellent extensions which are not Picard-Vessiot, for example by looking at subfields of Picard-Vessiot extensions. A slightly more subtle question is whether there are excellent extensions which are not subfields of Picard-Vessiot extensions. The answer is "no", as we will see in Corollary 14.

Applying condition 2 of Definition 2 requires that one be able to recognize field elements that satisfy linear differential equations. The following lemma is useful in that regard.

Lemma 2. Let $R \supseteq F$ be a differential algebra and let $\alpha \in R$. Then $\alpha$ satisfies $a$ linear differential equation over $F$ if and only if there is a $D_{R}$ stable finite dimensional $F$ subspace $V$ of $R$ containing $\alpha$. 
Proof. Suppose $L(\alpha)=0$ where $L=Y^{(n)}+a_{n-1} Y^{(n-1)}+\ldots+a_{1} Y^{(1)}+a_{0} Y$, so $\alpha^{(n)}=-\sum a_{i} \alpha^{(i)}$. Let $V$ be the $F$ span of $\alpha^{(i)}, 0 \leq i \leq n-1$. For $f \in F, D_{R}\left(f \alpha^{(i)}\right)=$ $D(f) \alpha^{(i))}+f \alpha^{(i+1)}$. When $i<n-1$, we clearly have $D_{R}\left(f \alpha^{(i)}\right) \in V$, and for $i=n-1$, $D_{R}\left(f \alpha^{(n-1)}\right)=D(f) \alpha^{(n-1)}-f \sum_{i<n} a_{i} \alpha^{(i)}$ which is in $V$ as well. It follows that $V$ is closed under $D_{R}$. If, conversely, $\alpha$ belongs to a $D_{R}$ stable subspace $V$ of $R$ of dimension $n$ over $F$, then the set $\left\{\alpha^{(i)} \mid 0 \leq i \leq n\right\}$ is necessarily linearly dependent over $F$, from which it follows that $\alpha$ satisfies a linear differential equation of order $n$ over $F$.

The definition of locally excellent asserts that a locally excellent field is the direct limit of excellent subfields. As the following proposition shows, it is sufficient that it be a union of excellent subfields.

Proposition 3. Let $E \supseteq F$ be a no new constants extension. Let $E_{e}$ be the union of all excellent subfields of $E$. Then $E_{e}$ is a locally excellent subfield of $E$, and $E$ is locally excellent if and only if $E=E_{e}$.

Proof. Let $\alpha_{1}$ and $\alpha_{2}$ belong to $E_{e}$. Suppose that $\alpha_{i} \in E_{i}$ where $E_{i} \supseteq F$ is excellent, and suppose that $E_{i}$ is the quotient field of the excellent integral domain $R_{i}$ where $F \subseteq R_{i} \subseteq E_{i}$. Consider the integral domain $R=R_{1} R_{2}=\left\{\sum \beta_{j} \gamma_{j} \mid \beta_{j} \in R_{1}, \gamma_{j} \in R_{2}\right\}$. It is clearly finitely generated over $F$, and its quotient field, being contained in $E$, has no new constants. Let $\sum \beta_{j} \gamma_{j}$ be an element of $R$, and let $V_{j}$ and $W_{j}$ be finite $F$ dimensional $D$ stable subspaces of $R_{1}$ and $R_{2}$ such that $\beta_{j} \in V_{j}$ and $\gamma_{j} \in W_{j}$ (these exist by Lemma 2). Then $V=\sum V_{j} W_{j}$ is a finite $F$ dimensional $D$ stable subspace of $R$ containing $\sum \beta_{j} \gamma_{j}$, so, by Lemma $2, \sum \beta_{j} \gamma_{j}$ satisfies a linear differential equation over $F$. It follows that $R$ is excellent, and hence so is its quotient field $E_{1} E_{2}$, so $E_{1} E_{2} \subseteq E_{e}$. Since $\alpha_{i} \in E_{i}$, we have the sum, product, and quotient (if defined) of the $\alpha_{i}$ in the excellent subfield $E_{1} E_{2}$ and hence belong to $E_{e}$ as well. It follows that $E_{e}$ is a field. If $\mathcal{F}=\left\{\alpha_{1}, \ldots, \alpha_{n}\right\}$ is a finite subset of $E_{e}$, with $\alpha_{i} \in E_{i}$ for some excellent subfield $E_{i}$ of $E$, then the above argument applied inductively shows that $E_{1} \cdot E_{2} \cdot \ldots \cdot E_{n}$ is an excellent subfield of $E_{e}$ containing $\mathcal{F}$, and it follows that $E_{e}$ is locally excellent. And of course if $E$ is locally excellent, it coincides, by definition, with $E_{e}$.

The set of solutions of a linear differential equation $L=0$ over $F$ in a no new constants extension $E$ of $F$ is a vector space over the constants $C$ of dimension at most the order of $L$. The cardinality of the set of equations over $F$ is that of $F$, and the cardinality of any finite dimensional $C$ space is that of $C$. It follows that the set of elements of the NNC extension $E \supseteq F$ that satisfy linear differential equations over $F$ has cardinality at most that of $F$, as would the set of their ratios. We conclude:

Lemma 4. Let $E \supseteq F$ be a no new constants extension. Let $E_{e}$ be the union of all excellent subfields of $E$. Then $E_{e}$ has cardinality that of $F$. In particular, the cardinality of an excellent or locally excellent extension of $F$ is that of $F$.

We are going to use Lemma 4 in a Zorn's Lemma argument to produce locally excellent extensions with certain maximality properties. The following Lemma covers the inductive step in those arguments: 
Lemma 5. Let $E$ be a simply excellent extension of $F$ and let $K$ be a NNC extension of $F$. Then there is a NNC extension $K_{1} \supseteq F$ and differential $F$ embeddings $E \rightarrow K_{1}$ and $K \rightarrow K_{1}$. If $K$ is locally excellent, $K_{1}$ may be taken to be locally excellent.

Proof. Suppose that $E$ is the quotient field of the differential integral domain $R$, where $R$ a differentially simple excellent $F$ algebra. Let $R_{1}$ be the finitely generated $K$ algebra $K \otimes_{F} R$, and let $P$ be a maximal differential ideal of $R_{1}$. By [2, Cor. 1.8, p. 11], $R_{1} / M$ is a differential integral domain whose quotient field $K_{1}$ is a NNC extension of $K$. Thus $K_{1}$ is a NNC extension of $F$. The map $x \mapsto x \otimes 1$ induces a differential $F$ embedding $K \rightarrow K_{1}$. The map $y \mapsto 1 \otimes y$ induces a differential $F$ algebra homomorphism $R \rightarrow K_{1}$; since $R$ is differentially simple this is an embedding which then extends to an $F$ embedding $E \rightarrow K_{1}$. Let $K_{e}$ denote the union of the excellent subfields of $K_{1}$. By Proposition $3, K_{e}$ is a subfield of $K_{1}$, and of course it contains the image of $E$ under the above embedding. If $K$ is locally excellent, then $K_{e}$ contains the image of $K$ under the above embedding and hence we can replace $K_{1}$ by $K_{e}$ in the conclusion of the lemma.

We now establish the main compositum result for excellent extensions:

THEOREM 6. There exist maximal locally excellent extensions $K$ of $F$. If $K$ is such, and if $E$ is any simply excellent extension of $F$ then $E$ can be differentially $F$ embedded into $K$.

Proof. Fix a set $Y \supset F$ of cardinality greater than that of $F$. Let $\mathcal{S}$ denote the set of locally excellent extensions of $F$ whose underlying set is a subset of $Y$. By Lemma 4 , any locally excellent extension of $F$ is $F$ differentially isomorphic to an element of $\mathcal{S}$. The union of any chain in $\mathcal{S}$ is a locally excellent extension of $F$ and, by Lemma 4 , again in $\mathcal{S}$. Hence by Zorn's Lemma $\mathcal{S}$ has maximal elements. Let $K$ be one, and let $E$ be a simply excellent extension of $F$. By Lemma 5 , there is a locally excellent extension $K_{1}$ of $F$ containing images of both $E$ and $K$. By transport of structure, we can assume that $K \subseteq K_{1}$. Then by using the fact that the cardinality of $K_{1}$ and $F$ are the same (Lemma 4) and that of $Y$ is larger, we can construct a field in $\mathcal{S}$ containing $K$ and isomorphic to $K_{1}$; we denote this field by $K_{1}$ as well. By maximality, $K=K_{1}$, and by construction of $K_{1}$, $E$ is $F$ differentially embedded in $K_{1}$, hence $K$.

Our most important use of the above construction is the following corollary:

Corollary 7. There is a no new constants extension of $F$ in which every PicardVessiot extension of $F$ embeds; any maximal locally excellent extension is such.

Proof. Picard-Vessiot extensions of $F$ are simply excellent, so any maximal locally excellent extension is, by Theorem 6 , a NNC extension with the desired property.

As another application of Theorem 6, we will deduce that the compositum of PicardVessiot extensions inside a no new constants extension is also Picard-Vessiot. The following lemmas isolate the main points of the argument:

Lemma 8. Let $R_{i}, i=1,2$ be simply excellent $F$ subalgebras of the no new constants extension $K \supseteq F$, and let $E_{i}$ denote the quotient field of $R_{i}$. Suppose that, for both $i=1$ and $i=2$, for every $N N C$ extension $M \supseteq F$ and every pair of differential $F$ embeddings 
$\phi, \psi: E_{i} \rightarrow M, \phi\left(E_{i}\right)=\psi\left(E_{i}\right)$. Then $S=R_{1} R_{2}$ is simply excellent, and if $E$ is its quotient field then all differential $F$ embeddings of $E$ in a given NNC extension have the same image.

Proof. Let $P$ be any prime differential ideal of $T=R_{1} \otimes_{F} R_{2}$ such that the quotient field of $T=\left(R_{1} \otimes_{F} R_{2}\right) / P$ has no new constants. It follows from Lemma 2 that every element of $T$ satisfies a linear homogeneous differential equation over $F$, and then it is clear that $T$ is excellent. Let $M$ be a maximal locally excellent extension of $F$. By Theorem 6 , the quotient fields of $S$ and $T$ both embed in $M$, as do $E_{1}$ and $E_{2}$. We suppose the later to be the inclusion, and then by the embedding images property of the $E_{i}$ we have that the compositum $E_{1} E_{2}$ is (isomorphic to) the quotient fields of both $S$ and $T$. In particular, both $S$ and $T$ have the same dimension, $m$, say, independent of the choice of $P$. We can choose $P=P_{0}$ to be the kernel of $R_{1} \otimes_{F} R_{2} \rightarrow S$, and then we can take $P=P_{1}$ to be a maximal differential ideal containing $P_{0}$. Since $R_{1} \otimes_{F} R_{2}$ modulo $P_{0}$ and $P_{1}$ both have dimension $m$, we conclude that $P_{0}=P_{1}$ is maximal differential and hence that $S=\left(R_{1} \otimes_{F} R_{2}\right) / P_{0}$ is simple. And it is clear that the quotient field $E=E_{1} E_{2}$ satisfies the embedding condition.

Lemma 9. Let $S$ be a simply excellent $F$ algebra with quotient field $E$, and suppose that all differential $F$ embeddings of $E$ in any given $N N C$ extension have the same image. Let $a \in E-F$. Then there is a differential $F$ algebra automorphism $\sigma$ of $E$ over $F$ with $\sigma(a) \neq a$. In particular, $E$ is a Picard-Vessiot extension of $F$.

Proof. The proof is essentially the same as [2, Thm. 3.7, p. 26]. There is an element $b \in S$ such that $a \in S\left[b^{-1}\right]$ (which is also differentially simple, finitely generated as an $F$ algebra, and has quotient field $E$ ), and we can replace $S$ with $S\left[b^{-1}\right]$ and assume that $a \in S$. By assumption, $c=a \otimes 1-1 \otimes a$ is not zero in the finitely generated $F$ algebra $S \otimes S$, and so we can find a maximal differential ideal $Q$ of $S \otimes S$ such that $c \notin Q$. Let $K$ be the quotient field of $(S \otimes S) / Q . K$ is a NNC extension of $F$. Since $S$ is differentially simple, the maps $S \rightarrow K$ induced from $x \mapsto(x \otimes 1)+Q$ and $x \mapsto(1 \otimes x)+Q$ are injective and hence induce differential $F$ embeddings from $E$ to $K$. These have the same image, and send $a$ to different things, so their ratio is the desired automorphism $\sigma$.

Now we revert to our original algebra $S$. Since $S$ is excellent, it is finitely generated over $F$, say by $x_{1}, \ldots, x_{k}$, and each $x_{i}$ is a solution of a linear homogeneous differential equation $L_{i}=0$ over $F$. Let $V_{i}$ be the set of all solutions of $L_{i}=0$ in $E$, and let $V=V_{1}+\ldots+V_{k} . V$ is stable under the group $G$ all differential $F$ automorphisms of $E$, and by the first part of the proof the fixed field of $G$ is $F$. Since $S$ is differentially simple, $E$ has no new constants over $F$, and then it follows (say by [2, Prop. 3.9, p. 27]) that $E$ is Picard-Vessiot over $F$.

It is now a trivial matter to show that the compositum of Picard-Vessiot extensions inside a no new constants extension is also Picard-Vessiot:

Corollary 10. Let $K$ be a no new constants extension of $F$ and let $E_{1}, \ldots, E_{k}$ be Picard-Vessiot subextensions of $F$ in $K$. Then the compositum $E_{1} \cdot E_{2} \cdot \ldots \cdot E_{k}$ is a Picard-Vessiot extension of $F$. 
Proof. By induction, it suffices to treat the case $k=2$. Then each $E_{i}$ is the quotient field of a simply excellent $R_{i}$ and $R_{1}, R_{2}$ satisfy the hypotheses of Lemma 8 . It follows from that lemma that $S=R_{1} R_{2}$ satisfies the hypotheses of Lemma 9 , and hence by that lemma the quotient field $E_{1} E_{2}$ of $S$ is a Picard-Vessiot extension of $F$ as desired.

When we apply Corollary 10 to the maximal locally excellent extensions of $F$, we get the following information about their structure:

TheOREM 11. Let $K_{m}$ be a maximal locally excellent extension of $F$. Then every finite subset of $K_{m}$ belongs to a Picard-Vessiot subextension. Thus $K_{m}$ is locally PicardVessiot. In particular, $K_{m}$ is the union of its Picard-Vessiot subextensions, and (a unique isomorphic copy) of every Picard-Vessiot extension of F occurs in $K_{m}$.

Proof. Every finite subset of $K_{m}$ belongs to an excellent subextension. Let $K$ be such, and suppose that $K$ is differentially generated over $F$ by elements $x_{1}, \ldots, x_{k}$ such that $x_{i}$ is a solution of the differential equation $L_{i}=0$. By Corollary 7, there are Picard-Vessiot extensions $E_{i}$ for $L_{i}$ inside $K$. Note that $x_{i} \in E_{i}$. By Corollary 10, the compositum $E$ of the $E_{i}$ is a Picard-Vessiot extension of $F$ in $K$ containing the $x_{i}$, and hence containing $K$. The final assertion is just Corollary 7 plus the fact that Picard-Vessiot extensions have unique images in NNC extensions [2, Prop. 3.3, p. 24].

We will see in the next section that it is a consequence of Theorem 11 that all maximal locally excellent extensions of $F$ are isomorphic.

We conclude this section with a few additional consequences of the above results. First, we note that we have established the existence of Picard-Vessiot extensions for (finite) sets of linear differential operators:

Proposition 12. Let $L_{1}, \ldots, L_{n}$ be monic homogeneous linear differential operators over F. Then there exists a Picard-Vessiot extension E of F which

1. contains a Picard-Vessiot extension for each $L_{i}$; and

2. is differentially generated over $F$ by the solution spaces $L^{-1}(0), 1 \leq i \leq n$.

Proof. Let $K$ be a maximal locally excellent extension of $F$. By Corollary 7 , there is a Picard-Vessiot extension $E_{i}$ of $F$ for $L_{i}$ in $K$. By Corollary $10, E=E_{1} \cdot \ldots \cdot E_{n}$ is a Picard-Vessiot extension of $F$ which satisfies the assertions of the proposition.

We can now show that excellent extensions embed in Picard-Vessiot extensions:

Proposition 13. Let $F\left\langle x_{1}, \ldots, x_{n}\right\rangle$ be a finitely generated $N N C$ differential field extension of $F$, and suppose for each $i$ that $x_{i}$ satisfies a monic linear homogeneous differential equation $L_{i}=0$ over $F$. Then $F\left\langle x_{1}, \ldots, x_{n}\right\rangle$ can be embedded in a Picard-Vessiot extension of $F$.

Proof. Let $M=F\left\langle x_{1}, \ldots, x_{n}\right\rangle$. By Proposition 12, there is a Picard-Vessiot extension $E_{0}$ of $M$ which contains a Picard-Vessiot extension of $M$ for each $L_{i}$. Let $V_{i} \subset E_{0}$ denote $L_{i}^{-1}(0)$; we note that $x_{i} \in V_{i}$. The differential subfield $E_{i}=F\left\langle V_{i}\right\rangle$ of $E_{0}$ generated over $F$ by $V_{i}$ is a Picard-Vessiot extension of $F$ for $V_{i}$, and by Corollary $10, E=E_{1} \cdot \ldots \cdot E_{n}$ is a Picard-Vessiot extension of $F$. Since all the $x_{i}$ belong to $E, M$ is contained in $E$. 
Among the consequences of Proposition 13, we have that excellent extensions are subextensions of Picard-Vessiot extensions:

COROLlary 14. Excellent extensions of F are subextensions of Picard-Vessiot extensions.

Proof. Since excellent extensions are differentially generated over $F$ by finitely many elements each of which satisfies a linear differential equation over $F$, this is a special case of Proposition 13.

3. Picard-Vessiot closures. As we saw in Section 2, maximal locally excellent extensions of $F$ are unions of their Picard-Vessiot subextensions, and every PicardVessiot extension occurs (up to isomorphism). That is, they are Picard-Vessiot closures of $F$. In this section, we will show that these extensions are unique (up to isomorphism), and that automorphisms of $F$ extend to them.

The maximal locally excellent extensions are constructed from a Zorn's Lemma/maximization method, and then seen to be generated by (actually a union of) representatives of the isomorphism classes of Picard-Vessiot extensions of $F$. Alternatively, one could start with representatives of all these isomorphism classes, form their (infinite) tensor product, pass to the quotient by a prime differential ideal, and take the quotient field of the result. This of course provides a differential field containing a representative of each isomorphism class of Picard-Vessiot extensions of $F$; the difficulty is to find such a field with no new constants. That this is possible at all will be seen to follow from Theorem 11, and we will further show that all such constructions yield isomorphic fields.

We begin by considering a similar construction with finitely many Picard-Vessiot extensions of $F$ :

Proposition 15. Let $E_{1}, \ldots, E_{n}$ be Picard-Vessiot extensions of $F$, and let $S=$ $E_{1} \otimes_{F} \ldots \otimes_{F} E_{n}$. Let $G_{i}=G\left(E_{i} / F\right)$ be the group of differential automorphisms of $E_{i}$ over $F$ and let $G=G_{1} \times \ldots \times G_{n}$. Regard $G$ as a group of automorphisms of $S$. Suppose that $P$ and $Q$ are prime differential $c N N C$ ideals of $S$. Then there is $g \in G$ such that $g(P)=Q$. The set of all such $g$ is a coset of a Zariski closed subgroup of $G$.

Proof. Let $K$ be the quotient field of $S / P$ and let $M$ be the quotient field of $S / Q$. Both $K$ and $M$ are NNC composita of $E_{1}, \ldots, E_{n}$, and hence themselves Picard-Vessiot by Corollary 10. Hence, by Theorem 11 both $K$ and $M$ can be embedded over $F$ in a maximal locally excellent extension $K_{m}$, say via $\sigma: K \rightarrow K_{m}$ and $\tau: M \rightarrow K_{m}$. We let $s: S \rightarrow K_{m}$ and $t: S \rightarrow K_{m}$ be the corresponding maps with respective kernels $P$ and $Q$ coming from $\sigma$ and $\tau$. We regard $E_{i}$ as a subring of $S$. The restrictions of $s$ and $t$ to $E_{i}$ are two embedings of $E_{i}$ in an NNC field, and hence their images coincide. It follows that there is $g_{i} \in G_{i}$ such that $s=t g_{i}$ on $E_{i}$. Let $g=\left(g_{1}, \ldots, g_{n}\right)$. Then $s=t \circ g$ on each $E_{i}$, and hence on $S$, and it follows that the kernel of $t$ is $g(\operatorname{Ker}(s))$. Since $s$ has kernel $P$ and $t$ has kernel $Q$, it follows that $g(P)=Q$.

Let $H \leq G$ be the stabilizer of $P$. The set of all elements of $G$ that carry $P$ to $Q$ is a coset of $H$, and hence it suffices to prove that $H$ is Zariski closed in $G$. Each $E_{i}$ is the quotient field of the simply excellent domain $R_{i}$ consisting of all elements of $E_{i}$ which 
satisfy linear differential equations over $F\left[2\right.$, Prop. 5.1, p. 61] and $G_{i}$ acts as an algebraic transformation group on $R_{i}$ ([2, p. 49]; indeed, $R_{i}$ can alternatively be characterized as the maximal such subspace of $E_{i}$ ). Let $R=R_{1} \otimes_{F} \ldots \otimes_{F} R_{n}$. G acts as an algebraic transformation group on $R$ as well. $S$ is a localization of $R$, and for $h \in G, h(P)=P$ if and only if $h(P \cap R)=P \cap R$. Hence it suffices to prove that the stabilizer of $P \cap R$ in $G$ is Zariski closed. $G$ is an algebraic group over $C$, and while $R$ is not a finitely generated $C$ algebra, it nonetheless is true that the stabilizer of an ideal of $R$ is closed in $G,[2, \mathrm{p}$. 49]. Hence $H$ is closed, as asserted.

It is now straightforward to generalize Proposition 15 to cover the infinite case we need:

TheOREM 16. Let $\left\{E_{i} \mid i \in \mathcal{I}\right\}$ be a set of Picard-Vessiot extensions of $F$, and let

$$
S=\bigotimes_{i \in \mathcal{I}} E_{i}
$$

Let $G_{i}=G\left(E_{i} / F\right)$ and let

$$
G=\prod_{i \in \mathcal{I}} G_{i}
$$

Regard $G$ as a group of automorphisms of $S$. Suppose that $P$ and $Q$ are $c N N C$ prime differential ideals of $S$. Then there is $g \in G$ with $g(P)=Q$.

Proof. Let $\mathcal{F}$ be a finite subset of $\mathcal{I}$. Let $S_{\mathcal{F}}=\bigotimes_{i \in \mathcal{F}} E_{i}$ and let $P_{\mathcal{F}}$ (respectively $Q_{\mathcal{F}}$ ) denote the intersection $P \cap S_{\mathcal{F}}$ (respectively $Q \cap S_{\mathcal{F}}$ ). Since $S_{\mathcal{F}} / P_{\mathcal{F}}$ injects into $S / P, P_{\mathcal{F}}$ is cNNC. Similarly, $Q_{\mathcal{F}}$ is cNNC. By Proposition 15, there is $g \in G_{\mathcal{F}}=\prod_{i \in \mathcal{F}} G_{i}$ such that $g\left(P_{\mathcal{F}}\right)=Q_{\mathcal{F}}$. In particular, the set

$$
X(\mathcal{F})=\left\{g \in G_{\mathcal{F}} \mid g\left(P_{\mathcal{F}}\right)=Q_{\mathcal{F}}\right\}
$$

is non-empty. We also note that $X(\mathcal{F})$ is the coset of a subgroup of $G_{\mathcal{F}}$; it is a coset of the stabilizer of $P$.

As $\mathcal{F}$ ranges over the finite subsets of $\mathcal{I}$, the groups $G_{\mathcal{F}}$ form an inverse system with $G=\lim G_{\mathcal{F}}$, and the sets $X(\mathcal{F})$ form a sub inverse system of cosets. The groups $G_{\mathcal{F}}$ are algebraic groups (over $C$ ), and, as we saw in Proposition 15, the cosets $X(\mathcal{F}$ ) are Zariski closed subsets. It then follows from [1, Prop. 2.7, p.504] that the inverse limit $X=\lim _{\longleftarrow} X(\mathcal{F})$ is non-empty. Clearly any element $g \in X$ satisfies $g(P)=Q$ as desired.

It is an immediate corollary of Theorem 16 that extensions of $F$ which are locally Picard-Vessiot are determined by the Picard-Vessiot extensions which occur in them. To make the notion of "occur in them" precise we formulate the following definition.

Definition 3. An isomorphism class $\mathcal{E}$ of Picard-Vessiot extensions of $F$ is said to occur in an extension $K$ of $F$ if there is a representative $E$ of $\mathcal{E}$ and an embedding $E \rightarrow K$ over $F$.

Theorem 17. Let $K_{1}$ and $K_{2}$ be locally Picard-Vessiot extensions of $F$. Then $K_{1}$ and $K_{2}$ are isomorphic over $F$ if and only if the isomorphism classes of Picard-Vessiot extensions which occur in each coincide. In particular, any two Picard-Vessiot closures of $F$ are isomorphic. 
Proof. Let $\left\{\mathcal{E}_{i} \mid j \in \mathcal{J}\right\}$ be the set of isomorphism classes of Picard-Vessiot extensions of $F$. For each $j \in \mathcal{J}$, let $E_{j}$ be a representative of $\mathcal{E}_{j}$. Let $\mathcal{J}\left(K_{i}\right)=\left\{j \in \mathcal{J} \mid \mathcal{E}_{j}\right.$ occurs in $K_{i}$ \}. There is an obvious surjection

$$
S_{i}=\bigotimes_{j \in \mathcal{J}\left(K_{i}\right)} E_{j} \rightarrow K_{i}
$$

whose kernel we denote $P_{i}$. If $\mathcal{J}\left(K_{1}\right)=\mathcal{J}\left(K_{2}\right)$, then $S_{1}=S_{2}$, which we will denote $S$. By Theorem 16, there is an automorphism $g$ of $S$ with $g\left(P_{1}\right)=P_{2} ; g$ then provides an isomorphism of $K_{1}$ with $K_{2}$. The reverse implication is obvious, and the main assertion of the theorem follows. If the $K_{i}$ are Picard-Vessiot closures, then $\mathcal{J}\left(K_{i}\right)=\mathcal{J}$ so the extensions occurring in both coincide.

We next want to see what happens to Picard-Vessiot closures when $F$ undergoes an automorphism. We begin this analysis by considering base change for Picard-Vessiot extensions.

Thus we assume that $E$ is a Picard-Vessiot extension of $F$ for $L=Y^{(n)}+a_{n-1} Y^{(n-1)}+$ $\ldots+a_{1} Y^{(1)}+a_{0} Y$, that $R$ is the simply excellent ring with quotient field $E$ consisting of the solutions in $E$ of linear differential equations over $F$, and that $\sigma: F \rightarrow K$ is a differential morphism from $F$ to a differential field with field of constants $C$, where $\sigma$ is the identity on $C$. We consider the differential $K$ algebra $S=K \otimes_{F} R$. The derivation $D_{S}$ satisfies $D_{S}(k \otimes r)=D_{K}(k) \otimes r+k \otimes D_{E}(r)$, and for $a \in F, k \sigma(a) \otimes r=k \otimes a r$. Hence if $y \in E$ satisfies $L(y)=0$, we have

$$
\begin{aligned}
0 & =1 \otimes\left(y^{(n)}+a_{n-1} y^{(n-1)}+\ldots+a_{1} y^{(1)}+a_{0} y\right) \\
& =\left(1 \otimes y^{(n)}\right)+\left(\sigma\left(a_{n-1}\right) \otimes y^{(n-1)}\right)+\ldots+\left(\sigma\left(a_{1}\right) \otimes y^{(1)}\right)+\left(\sigma\left(a_{0}\right) \otimes y\right) \\
& =z^{(n)}+\sigma\left(a_{n-1}\right) z^{(n-1)}+\ldots+\sigma\left(a_{1}\right) z^{(1)}+\sigma\left(a_{0}\right) z
\end{aligned}
$$

where

$$
z=1 \otimes y
$$

Thus $z$ is a solution of the equation

$$
\sigma(L)=0
$$

where $\sigma(L)$ is the operator

$$
L=Y^{(n)}+\sigma\left(a_{n-1}\right) Y^{(n-1)}+\ldots+\sigma\left(a_{1}\right) Y^{(1)}+\sigma\left(a_{0}\right) Y
$$

over $K$.

If $\left\{y_{1}, \ldots, y_{n}\right\}$ is a full set of solutions of $L=0$ in $E$, with Wronskian $w$ (which is a unit in $R$ ), then $z_{i}=1 \otimes y_{i}, 1 \leq i \leq n$, have Wronskian $1 \otimes w$, which is a unit in $S$. And $S$ is generated as a $K$ algebra by the $z_{i}$. Let $P$ be a maximal differential ideal of $S$, and let $M$ be the quotient field of $S / P$. Then $M$ is a NNC extension of $K$ and a Picard-Vessiot extension of $K$ for $\sigma(L)$. The morphism $R \rightarrow S \rightarrow S / P \rightarrow M$ induced by $r \mapsto 1 \otimes r$ is a non-trivial differential homomorphism, and hence injective since $R$ has no non-trivial differential ideals, and so extends to a differential morphism $\widehat{\sigma}: E \rightarrow M$ (the notation is chosen because $\widehat{\sigma}(a)=\sigma(a)$ for $a \in F)$. And, by construction, we have that $M=K \widehat{\sigma}(E)$. We record these remarks in the following proposition: 
Proposition 18. Let $E \supset F$ be a Picard-Vessiot extension for $L=Y^{(n)}+a_{n-1} Y^{(n-1)}$ $+\ldots+a_{0} Y$, let $K$ be a differential field with field of constants $C$ and let $\sigma: F \rightarrow K$ be a differential morphism. Let $M \supset K$ be a Picard-Vessiot extension for $\sigma(L)=Y^{(n)}+$ $\sigma\left(a_{n-1}\right) Y^{(n-1)}+\ldots+\sigma\left(a_{0}\right) Y$. Then there is a morphism $\widehat{\sigma}: E \rightarrow M$ extending $\sigma$ and such that $M$ is generated as a field over $K$ by $\widehat{\sigma}(E)$.

In particular, if $K=F$ and $\sigma$ is a differential automorphism of $F$ then $\widehat{\sigma}$ is a differential isomorphism from $E$ to $M$. Further, $F \otimes_{\sigma} E \rightarrow M$ by $a \otimes e \mapsto \sigma(a) \widehat{\sigma}(e)$ is an isomorphism, which we will also denote by $\widehat{\sigma}$. Thus $F \otimes_{\sigma} E$ is a Picard-Vessiot extension of $F$ and every Picard-Vessiot extension of $F$ is of this form.

Proof. All the assertions have been noted except the ones of the final paragraph. When $K=F, K=\sigma(F)$ is contained in $\widehat{\sigma}(E)$ so that the latter equals $M$, and thus $\widehat{\sigma}$ is onto as well as injective. Moreover, every element of $F \otimes_{\sigma} E$ can be written in the form $1 \otimes e$, and if such an element is in the kernel of $F \otimes_{\sigma} E \rightarrow M$ then $1 \widehat{\sigma}(e)=0$, so $e$ and $1 \otimes e$ are zero as well. Thus $F \otimes_{\sigma} E \rightarrow M$ is injective; it is obviously surjective and hence an isomorphism. It follows that $F \otimes_{\sigma} E$ is a field and a NNC extension of $F$. In the notation of the construction which preceded the proposition, $F \otimes_{\sigma} E$ is then the quotient field of the differential integral domain $S=F \otimes_{\sigma} R$, and it follows that $F \otimes_{\sigma} E$ is a Picard-Vessiot extension of $F$ (for $\sigma(L)$ ). If we start with an arbitrary monic linear homogeneous operator, apply $\sigma^{-1}$, take a Picard-Vessiot extension for the transformed operator and then tensor with $F$ via $\sigma$, we thus obtain a Picard-Vessiot extension for the original operator.

We retain the notation of the final paragraph of Proposition 18, and assume further that we have two differential operators $L_{1}$ and $L_{2}$, and corresponding Picard-Vessiot extensions $E_{1}, E_{2}$ and $M_{1}, M_{2}$. The isomorphisms $\widehat{\sigma}_{i}: F \otimes_{\sigma} E_{i} \rightarrow M_{i}$ are not $F$ linear. They do, however, fit into the commutative diagrams

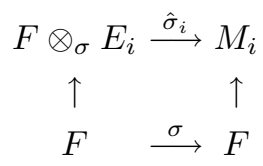

It follows that there is a well defined map

$$
\widehat{\sigma}_{1} \otimes \widehat{\sigma}_{2}:\left(F \otimes_{\sigma} E_{1}\right) \otimes_{F}\left(F \otimes_{\sigma} E_{2}\right) \rightarrow M_{1} \otimes_{F} M_{2}
$$

which is $\sigma$ semilinear as well.

Now we fix some notation:

Notation 1. Let $\mathcal{L}$ be the set of all monic linear homogeneous differential operators over $F$. For each $L \in \mathcal{L}$ let $E(L)$ be a Picard-Vessiot extension of $F$ for $L$. Fix a differential automorphism $\sigma$ of $F$. For each $L$ choose an isomorphism $\sigma_{L}: E(L) \rightarrow E(\sigma(L))$ as in Proposition 18; denote the corresponding isomorphism $F \otimes_{\sigma} E(L) \rightarrow E\left(\sigma(L)\right.$ by $\sigma_{L}$ as well. Let $\tau^{\sigma}$ be the (differential) isomorphism

given by

$$
T^{\sigma}=\bigotimes_{F}\left(F \otimes_{\sigma} E(L)\right)_{L \in \mathcal{L}} \rightarrow T_{\sigma}=\bigotimes_{F}(E(\sigma(L)))_{L \in \mathcal{L}}
$$

$$
\tau^{\sigma}=\bigotimes_{F}\left(\sigma_{L}\right)_{L \in \mathcal{L}}
$$


We follow Notation 1. $T^{\sigma}$ is a tensor product of $F$ algebras and hence an $F$ algebra. $T_{\sigma}$ is also an $F$ algebra. But $\tau^{\sigma}$ is not an $F$ algebra homomorphism, although it is $\sigma$ semilinear: $\tau^{\sigma}(a x)=\sigma(a) \tau^{\sigma}(x)$ for $a \in F$ and $x \in T^{\sigma}$. Let $K_{m}$ be a Picard-Vessiot closure of $F$. Of course $\{\sigma(L) \mid L \in \mathcal{L}\}=\mathcal{L}$. There is thus a surjection $\bigotimes_{F}(E(\sigma(L)))_{L \in \mathcal{L}} \rightarrow K_{m}$; let $P$ be its kernel and let $Q=\tau^{\sigma-1}(P)$. We have a differential isomorphism

$$
\overline{\tau^{\sigma}}: T^{\sigma} / Q \rightarrow T_{\sigma} / P
$$

induced from $\tau^{\sigma}$. Both $T^{\sigma} / Q$ and $T_{\sigma} / P$ are $F$ algebras, and, as before, although $\overline{\tau^{\sigma}}$ is not $F$ linear it is $\sigma$ semi-linear. Of course $T_{\sigma} / P$, being isomorphic to $K_{m}$, is a Picard-Vessiot closure of $F$. We claim that $T^{\sigma} / Q$ is as well. First, since $\overline{\tau^{\sigma}}$ is a differential isomorphism, the constants of $T^{\sigma} / Q$ coincide with those of $T_{\sigma} / P$, namely $C$, and hence $T^{\sigma} / Q$ is a NNC extension of $F$. Next, suppose that $E$ is any Picard-Vessiot extension of $F$. By Proposition $18, E$ is of the form $F \otimes_{\sigma} E(L)$ for some $L \in \mathcal{L}$. The natural map $F \otimes_{\sigma} E(L) \rightarrow T^{\sigma}$ then passes to an embedding of $F \otimes_{\sigma} E(L)$ into $T^{\sigma} / Q$, so that the isomorphism class of $E$ occurs in $T^{\sigma} / Q$. Since $T^{\sigma}$ is generated by Picard-Vessiot extensions (the $F \otimes_{\sigma} E(L)$ ), so is $T^{\sigma} / Q$, and since every isomorphism class occurs it follows easily that $T^{\sigma} / Q$ is a Picard-Vessiot closure of $F$ (and hence isomorphic to $K_{m}$ ). We conclude:

TheOrem 19. Let $\sigma$ be a differential automorphism of $F$ and let $K$ be a PicardVessiot closure of $F$. Then there is an automorphism $\bar{\sigma}$ of $K$ extending $\sigma$ on $F$.

Proof. In the notation of the discussion preceding the theorem, with $K_{m}=K, \bar{\sigma}$ is the composition of $\overline{\tau^{\sigma}}$ with the isomorphisms $K_{m} \simeq T^{\sigma} / Q$ and $T_{\sigma} / P \simeq K_{m}$.

Example 2. As we saw in Example 1 above, the Picard-Vessiot closure of $\mathbb{C}$ is the subfield $K=\mathbb{C}(t)(\{\exp (c t) \mid c \in \mathbb{C}\})$ of the power series field $\mathbb{C}((t))$. An automorphism $\sigma$ of $\mathbb{C}$ extends to $\mathbb{C}((t))$ by acting on coefficients; this action leaves $t$ fixed and carries $\exp (c t)=\sum(c t)^{k} / k !$ to $\exp (\sigma(c) t)$ and hence preserves $K$.

4. The tower of closures. It may happen that a Picard-Vessiot closure $K_{1} \supset F$ has proper Picard-Vessiot extensions, and hence $K_{1}$ has a proper Picard-Vessiot closure $K_{2}$. For example, if $F=\mathbb{C}(t)$, then $\log (t)$ belongs to the Picard-Vessiot closure $K$ of $F$, and $K(\log (\log (t))) \supset K$ is a proper Picard-Vessiot extension [3, p. 12-13]. And this process may continue. We introduce the following notation for these "higher PicardVessiot closures":

Notation 2. Let $K_{0}$ denote the base differential field $F$. We inductively define fields $K_{i}$ as follows: if $K_{i}$ has a proper Picard-Vessiot extension, then $K_{i+1} \supset K_{i}$ is a PicardVessiot closure of $K_{i}$. (The chain $K_{0} \subset K_{1} \subset \ldots$ may be finite or infinite.) Let $K_{\infty}$ denote the union of the chain whether it is finite or infinite. It is clear that $K_{\infty}$ is a field. We let $G_{i}, i \leq \infty$, denote the group of differential automorphisms of $K_{i}$ over $F$.

The field $K_{\infty}$ has no proper Picard-Vessiot extensions. This is trivial when the chain is finite, and when it is infinite, then we use the fact that any linear differential operator $L$ over $K_{\infty}$ has coefficients in $K_{i}$ for some $i$, and hence $K_{i+1}$ contains a Picard-Vessiot extension for $L$, namely a full set of solutions for $L$. But then so does $K_{\infty}$. Next, we record the behavior of the groups $G_{i}$ under the restriction of domain from $K_{i}$ to $K_{i+1}$ : 
Lemma 20. Let $\sigma \in G_{i}, i<\infty$. Then $\sigma\left(K_{j}\right)=K_{j}$ for all $j \leq i$. In particular, for $i<\infty$, under the restriction of domain from $K_{i}$ to $K_{j}$ for $i \geq j, G_{i}$ maps to $G_{j}$. Moreover, the restriction map $p_{i, j}: G_{i} \rightarrow G_{j}$ is surjective. $G_{\infty}$ is naturally identified with $\lim G_{i}$, with the projection maps $p_{i}: G_{\infty} \rightarrow G_{i}$ surjective and given by restriction.

Proof. By definition, $\sigma\left(K_{0}\right)=K_{0}$. Suppose we have shown that $\sigma\left(K_{i}\right)=K_{i}$, and let $a \in K_{i+1}$. Then $a$ belongs to a Picard-Vessiot extension $E$ of $K_{i}$ for some operator $L$ with coefficients in $K_{i}$. It then follows that $\sigma(E)$ is a Picard-Vessiot extension of $K_{i}$ for $\sigma(L)$. Hence $\sigma(E)$ is contained in $K_{i+1}$, so that $\sigma(a) \in K_{i+1}$. This proves that the maps $G_{i} \rightarrow G_{j}$ for $j<i<\infty$ are well defined. The maps $G_{i+1} \rightarrow G_{i}$ are surjective by Theorem 19 , and then all their composites are as well, proving surjectivity of $p_{i, j}$ for $i \geq j$. The final assertions are then standard results about countable (or finite) inverse limits.

Corollary 21. For $i<\infty$, the kernel of $G_{i+1} \rightarrow G_{i}$ is proaffine proalgebraic. Thus $\{e\}=\operatorname{Ker}\left(p_{i, i}\right) \subset \operatorname{Ker}\left(p_{i, i-1}\right) \ldots \subset \operatorname{Ker}\left(p_{i, 0}\right)=G_{i}$ is a normal series for $G_{i}$ with proaffine proalgebraic layers.

Proof. The kernel of the surjective map $G_{i+1} \rightarrow G_{i}$ is $A u t_{K_{i}}\left(K_{i+1}\right)$, which is proaffine proalgebraic.

The groups $G_{i}$ need not themselves, however, be proalgebraic. We will understand this through the following example:

We will consider the subfield of $\mathbb{C}((t))$ generated by $t$ and the series $\{\log (t+c) \mid c \in \mathbb{C}\}$. It is somewhat more convenient to consider this field abstractly as the pure transcendental field $K=\mathbb{C}(t)\left(\left\{y_{c} \mid c \in \mathbb{C}\right\}\right)$ with the derivation $D(t)=1$ and $D\left(y_{c}\right)=\frac{1}{y+c}$. We regard $K$ as an extension of $\mathbb{C}$ and of $\mathbb{C}(t)$. In the latter guise, it is a compositum of the Picard-Vessiot extensions $\mathbb{C}(t)\left(y_{c}\right) \supset \mathbb{C}(t)$, and hence locally Picard-Vessiot. Moreover, any differential automorphism of $K$ over $\mathbb{C}$ is seen to carry $t$ to $t$ plus an element of $\mathbb{C}$. Let $G$ be the group of differential automorphisms of $K$ over $\mathbb{C}$, let $H$ the group of differential automorphisms of $K$ over $\mathbb{C}(t)$, and let $C$ be the group of differential automorphisms of $\mathbb{C}(t)$ over $\mathbb{C}$.

We know that $H$ is a proaffine proalgebraic group. In fact it is easy to identify: any automorphism $\delta \in H$ carries each $y_{c}$ to a translate by a constant, say $\delta\left(y_{c}\right)=y_{c}+\delta(c)$. Conversely, any sequence of constants $\left\{d_{c}\right\}_{c \in \mathbb{C}}$ gives rise to an automorphism of $K$ over $\mathbb{C}(t)$ via the rule $y_{c} \mapsto y_{c}+d_{c}$. This correspondence $\delta \mapsto\{\delta(c)\}$ identifies $H$ with the product $\prod_{c \in \mathbb{C}} \mathbb{C}$ which we can regard as a proaffine proalgebraic group with coordinate ring $\mathbb{C}\left[\left\{Y_{c} \mid c \in \mathbb{C}\right\}\right]$.

The group $C$ is $\mathbb{C}$ acting via $t \mapsto t+\alpha$. For $\gamma \in \mathbb{C}$, we define the $\mathbb{C}$ automorphism $\sigma_{\gamma}$ of $K$ by $t \mapsto t+\gamma$ and $y_{c} \mapsto y_{c+\gamma}$. It is easily checked that $\sigma_{\gamma}$ is a differential automorphism of $K$ over $\mathbb{C}$, which restricts to translation by $\gamma$ on $\mathbb{C}(t)$. In particular, the restriction maps induces a surjection $G \rightarrow C$ (with kernel $H$ ). Let $\tau$ be any differential automorphism of $K$ over $\mathbb{C}$. Since $\tau(t)=t-\beta$ for some $\beta \in \mathbb{C}, \tau \sigma_{\beta}$ fixes $t$ and hence lies in $H$. It follows that $G$ is the semidirect product $H \rtimes C$ (where the latter sits inside $G$ via $\gamma \mapsto \sigma_{\gamma}$ ). The action of $C$ on $H$ is given by $\sigma_{c}^{-1} \delta \sigma_{c}$, which when composed with the correspondence $\delta \mapsto\{\delta(c)\}$ ends up being the translation action on the index in $\prod_{c \in \mathbb{C}} \mathbb{C}$, namely $\left\{\alpha_{c}\right\}_{c} \mapsto\left\{\alpha_{c}\right\}_{c+\gamma}$. 
We note that this semidirect product structure does not make $G$ into a proaffine proalgebraic group, despite the fact that both $H$ and $C$ are. The action of $C$ on $H$ on the coordinates $Y_{c}$ is via $Y_{c} \mapsto Y_{c+\delta}$. If $G$ were a proaffine proalgebraic group compatible with the semidirect product structure, then $Y_{c}$ would lie in the coordinate ring of $G$, but we have just seen that its $C$ translates (and hence its $G$ translates) span an infinite dimensional space over $\mathbb{C}$, which is a contradiction. If $G$ were a proaffine proalgebraic group under any structure compatible with those of its subgroup $H$ and its quotient $C$, then $G$ would be prounipotent and hence a semidirect product (since $C$ is free prounipotent on one generator), and a similar argument would lead to the same contradiction.

The tower of fields $K \supset \mathbb{C}(t) \supset \mathbb{C}$ is not part of the tower of Picard-Vessiot closures of $\mathbb{C}$, of course. It can, however, be embedded in that tower. We recall from Example 1 that $K_{1}=\mathbb{C}(t)(\{\exp (c t) \mid c \in \mathbb{C}\}) \subset \mathbb{C}((t))$ is a Picard-Vessiot closure of $\mathbb{C}$. The field $K=\mathbb{C}(t)\left(\left\{y_{c} \mid c \in \mathbb{C}\right\}\right)$ can be embedded into $\mathbb{C}((t))$ as well, via $t \mapsto t$ and $y_{c} \mapsto \log (t+c)$. We identify $K$ with its image.

We also want to consider the field $K_{1.5}=\mathbb{C}(t)(\{\exp (c t), \log (t+c) \mid c \in \mathbb{C}\}) \subset \mathbb{C}((t))$. By construction, $K_{1.5}$ contains the Picard-Vessiot closure $K_{1}$ of $\mathbb{C}$, and since $K_{1.5}$ is a compositum of Picard-Vessiot extensions of $\mathbb{C}(t)$, it is contained in a Picard-Vessiot closure $K_{2}$ of $K_{1}$. Since $K \subset K_{1.5}$, we have $K \subset K_{2}$.

We further claim that any differential automorphism $\sigma$ of $K_{2}$ fixing $\mathbb{C}$ pointwise preserves $K$ setwise. Since $D(\sigma(t))=1$, we must have $\sigma(t)=t+a$ for some $a \in \mathbb{C}$. Since $D\left(\sigma(\log (t+c))=\sigma\left(D(\log (t+c))=\frac{1}{t+c+a}\right.\right.$, we have $\sigma(\log (t+c))=\log (t+c)+d(c)$ for some $d(c) \in \mathbb{C}$. It follows that $\sigma(K) \subseteq K$. Note that in passing we have also remarked that $\sigma(\mathbb{C}(t)) \subseteq \mathbb{C}(t)$.

Now suppose that $G_{2}=\operatorname{Aut}_{\mathbb{C}}\left(K_{2}\right)$ has a proalgebraic structure such that $\operatorname{Aut}_{K_{1}}\left(K_{2}\right)$ is Zariski closed and the quotient isomorphism with $\operatorname{Aut}_{\mathbb{C}}\left(K_{1}\right)$ is a morphism of proalgebraic groups. Suppose further that for any set of elements in $K_{2}$ their fixer in $G_{2}$ is Zariski closed. Then $\operatorname{Aut}_{K}\left(K_{2}\right)$ is closed and the quotient of $G_{2}$ by it maps injectively under restriction to $G=\operatorname{Aut}_{\mathbb{C}}(K)$. Using the semidirect structure of $G$ above, one can see that this injection is an isomorphism. But since $G$ is not a proalgebraic group, neither is the quotient, and hence there is no such structure on $G_{2}$.

We revert to the general case and close this section with some comments: the nature of the groups $G_{i}$ and $G_{\infty}$ remains a mystery. Obviously the partial proalgebraic group structure they exhibit is important, but exactly what that structure is, and exactly what groups with that structure can occur, is in need of further research and clarification.

\section{References}

[1] G. Hochschild and G. D. Mostow, Representations and representative functions of Lie groups, Ann. of Math. 66 (1957), 495-542.

[2] A. Magid, Lectures on Differential Galois Theory, University Lecture Series 7, Amer. Math. Soc., Providence RI, 1997 (second printing with corrections).

[3] A. Magid, The Picard-Vessiot antiderivative closure, J. Algebra 244 (2001), 1-18.

[4] M. van der Put and M. Singer, Differential Galois theory (to appear), see http://www4. ncsu.edu/ singer/ms_papers.html. 